\title{
The validity of modulation equations for extended systems with cubic nonlinearities
}

\author{
Pius Kirmann, Guido Schneider and Alexander Mielke \\ Mathematisches Institut A, Universität Stuttgart, Pfaffenwaldring 57, \\ W-7000 Stuttgart, Germany
}

(MS received 3 October 1991)

\section{Synopsis}

Modulation equations play an essential role in the understanding of complicated systems near the threshold of instability. Here we show that the modulation equation dominates the dynamies of the full problem locally, at least over a long time-scale. For systems with no quadratic interaction term, we develop a method which is much simpler than previous ones. It involves a careful bookkeeping of errors and an estimate of Gronwall type.

As an example for the dissipative case, we find that the Ginzburg-Landau equation is the modulation equation for the Swift-Hohenberg problem. Moreover, the method also enables us to handle hyperbolic problems: the nonlinear Schrödinger equation is shown to describe the modulation of wave packets in the Sine-Gordon equation

\section{Introduction}

We consider scalar evolutionary problems on the real line. In the parabolic case, we are interested in the behaviour of systems close to the threshold of instability. If a spatially homogeneous solution becomes unstable, typically a whole band of wave numbers turns unstable. In this situation, the bifurcating solutions can be approximately described by a so-called amplitude or modulation equation $([3,8,9])$. As an example, we study the (scalar) Swift-Hohenberg equation ([2]):

$$
\partial_{t} u(t, x)=L_{\lambda}\left(\partial_{x}\right) u(t, x)-u^{3}(t, x), \quad \text { with } \quad L_{\lambda}\left(\partial_{x}\right) u=-\left(1+\partial_{x}^{2}\right)^{2} u+\lambda u \text {. }
$$

The trivial solution $u \equiv 0$ is unstable for $\lambda>0$ and, linearising at $u=0$, we find solutions of the form $u(t, x)=e^{\mu t+t k x}$, where $\mu(k)=-\left(1-k^{2}\right)^{2}+\lambda$ is positive for $k$ close to \pm 1 . One expects that for small $\lambda>0$ there are solutions which are slow modulations in time and space of the critical modes $e^{ \pm x x}$. Using the scalings $\lambda=\varepsilon^{2}, T=\varepsilon^{2} t$ and $X=\varepsilon x$, we introduce the formal approximation

$$
u_{A}(t, x)=\varepsilon\left(A(T, X) e^{i x}+\bar{A}(T, X) e^{-\iota x}\right) .
$$

Substituting this ansatz into (1.1) and equating the coefficients of $e^{i x}$ of order $O\left(\varepsilon^{3}\right)$ to zero, we find that the amplitude $A$ has to satisfy the Ginzburg-Landau equation

$$
\partial_{T} A=4 \partial_{X}^{2} A+A-3|A|^{2} A .
$$

Now taking a solution $A$, the question arises as to how well $u_{A}$ approximates the solution $u(t, x)$ of the original problem which has the same initial data.

This question was treated in [2] for the Swift-Hohenberg problem and in [11] for general scalar equations with quadratic nonlinearities. The result obtained 
there implies that $u_{A}$ approximates the solution $u$ of the original problem up to an error of order $O\left(\varepsilon^{2}\right)$, uniformly for all $(t, x) \in\left[0, T_{0} / \varepsilon^{2}\right] \times \mathbb{R}$. Yet both studies lead to very involved analyses. Here we want to show that it is possible to obtain the same results by a much simpler method, which, however, is restricted to the case where the nonlinearity starts with cubic terms. This is, for instance, always the case for problems with odd nonlinearities. Our method consists in finding a good approximate solution $v_{A}$ which is typically one order more accurate than $u_{A}$. Then we estimate the error $R=\left(u-v_{A}\right) / \varepsilon^{2}$ directly from the equation which is found by substituting $u=v_{A}+\varepsilon^{2} R$ into the full problem. The essential ingredients of the method are the boundedness of the semigroup generated by the linearised problem (at criticality) and the relative smallness of the nonlinear terms. In particular, the perturbation to the linear part is of order $\varepsilon^{2}$, which allows estimates over the time scale $1 / \varepsilon^{2}$.

In contrast to the previous work, our analysis does not rely on the smoothing properties of the linearised flow. Hence, we are also able to treat hyperbolic problems. The formal derivation of amplitude equations for hyperbolic problems is discussed extensively in [1], but rigorous approximation results in the sense mentioned above are rare, see e.g. [5], §6. However, for problems on bounded $x$-domains, the theory of averaging was applied to show that finite mode (Galerkin) truncations lead to good approximations over long time-scales, see $[7,10]$. In the case of an unbounded $x$-domain, we are interested in the evolution of slowly modulated wave packets. For the Sine-Gordon equation $\partial_{x}^{2} u=\partial_{x}^{2} u-$ $\sin u$, we prove that solutions of the type

$u_{A}(t, x)=\varepsilon\left(A\left(\varepsilon^{2} t, \varepsilon(x-v t)\right) e^{t(k x-\omega t)}+\right.$ c.c. $)$, where $\omega^{2}=k^{2}+1, v=k / \omega$,

are $O\left(\varepsilon^{3 / 2}\right)$-approximations (in the $L_{2}(\mathbb{R})$-norm) over the time scale $1 / \varepsilon^{2}$ of an exact solution $u$. Here $A=A(T, X)$ has to be a solution of the nonlinear Schrödinger equation

$$
2 i \omega \partial_{T} A=\left(v^{2}-1\right) \partial_{X}^{2} A+\frac{1}{2}|A|^{2} A .
$$

The simplicity of the method makes the theory amenable to several generalisations discussed in Section 4. For instance, one can treat vector-valued $u$, even with values in an infinite-dimensional Banach space, allowing for applications to problems on cylindrical domains.

\section{The parabolic case}

We prove the following approximation result for the Swift-Hohenberg problem (SHE) (1.1) through the Ginzburg-Landau equation (GLE) (1.3). It is shown in [2, Lemma 3.1] that (1.3) has for each initial datum $A(0, X) \in C_{b}^{4}(\mathbb{R})$ a unique solution which is defined for all $T>0$ and bounded in $C_{b}^{4}(\mathbb{R})$. The same statement holds for the SHE. (Here $C_{b}(\mathbb{R})$ denotes the space of bounded and uniformly continuous functions.)

THEOREM 2.1. Let $A=A(T, X)$ be a solution of the GLE and $u_{A}$ the formal approximation (1.2). Then, for each $T_{0}>0$ and $d>0$, there exist $\varepsilon_{0}, C>0$ such that for all $\varepsilon \in\left(0, \varepsilon_{0}\right)$ the following statement holds. Let $u=u(t, x)$ be a solution of 
the SHE such that $\left|u(0, x)-u_{A}(0, x)\right| \leqq d \varepsilon^{2} ;$ then the estimate

$$
\left|u(t, x)-u_{A}(t, x)\right|<C \varepsilon^{2}, \text { for all }(t, x) \in\left[0, T_{0} / \varepsilon^{2}\right] \times \mathbb{R},
$$

is satisfied.

Remarks 2.2. Note that $u_{A}$, and hence $u$, are $O(\varepsilon)$ and that the error is one order smaller. The approximation occurs on the natural time-scale $O\left(1 / \varepsilon^{2}\right)$ for the modulations. Taking solutions $u_{1}$ and $u_{2}$ with two initial conditions $O\left(\varepsilon^{2}\right)$-close to $u_{A}(0, \cdot)$ we find, by using (2.1) and the triangle inequality, that $u_{1}$ and $u_{2}$ stay $O\left(\varepsilon^{2}\right)$-close over the time interval $\left[0, T_{0} / \varepsilon^{2}\right]$.

Proof of Theorem 2.1. We want to show that the error $u(t, x)-u_{A}(t, x)$ remains of order $O\left(\varepsilon^{2}\right)$ over the time $t \leqq T_{0} / \varepsilon^{2}$. However, substituting $u_{A}$ into (1.1) leaves the residual term $\varepsilon^{3} A^{3} e^{3 x x}$ (cf. [2]) which, upon integration over $\left[0, T_{0} / \varepsilon^{2}\right]$, leads to an error $O(\varepsilon)$. To avoid this difficulty, we use an improved approximation:

$$
v_{A}(t, x)=\varepsilon A(T, X) e^{2 x}-\varepsilon^{3} \frac{1}{64} A(T, X)^{3} e^{3 \ell x}+\text { c.c. }
$$

Using the relation

$$
\begin{aligned}
- & L_{0}\left(\partial_{x}\right)\left(B(\varepsilon x) e^{n x x}\right) \\
& =\left[\left(1-n^{2}\right)^{2} B+\varepsilon 4 i n\left(1-n^{2}\right) B^{\prime}+\varepsilon^{2}\left(2-6 n^{2}\right) B^{\prime \prime}+\varepsilon^{3} 4 i n B^{\prime \prime \prime}+\varepsilon^{4} B^{\prime \prime \prime \prime}\right] e^{n x x},
\end{aligned}
$$

we find the residuum

$$
\begin{aligned}
\rho(\varepsilon, t, x)= & \partial_{1} v_{A}-L_{0} v_{A}-\varepsilon^{2} v_{A}+v_{A}^{3} \\
= & \varepsilon^{3}\left[\left(\partial_{T} A-4 \partial_{X}^{2} A-A\right) e^{i x}-\left(1-3^{2}\right)^{2} \frac{1}{A_{A}} A^{3} e^{3 t x}+\text { c.c. }\right] \\
& +\varepsilon^{3}\left(A e^{2 x}+\bar{A} e^{-i x}\right)^{3}+O\left(\varepsilon^{4}\right) .
\end{aligned}
$$

Since $A$ solves the GLE, the whole $O\left(\varepsilon^{3}\right)$-term vanishes.

We let $\varepsilon^{2} R(t, x)=u(t, x)-v_{A}(t, x)$ and obtain

$$
\begin{aligned}
\partial_{t} R & =L_{0} R+\varepsilon^{2} a(\varepsilon, t, x) R+\varepsilon^{3} N(\varepsilon, t, x, R)+\varepsilon^{2} r(\varepsilon, t, x), \\
R(0, x) & =\left(u(0, x)-v_{A}(0, x)\right) / \varepsilon^{2} .
\end{aligned}
$$

where $a(\varepsilon, t, x)=1-3\left(v_{A}(t, x) / \varepsilon\right)^{2}, N(\varepsilon, t, x, R)=-3\left(v_{A} / \varepsilon\right) R^{2}-\varepsilon R^{3}, r=\rho / \varepsilon^{4}$ is bounded over $\left(0, \varepsilon_{0}\right) \times[0, x) \times \mathbb{R}$, and $|R(0, x)| \leqq 2 d$ for sufficiently small $\varepsilon_{0}$.

We solve this equation by turning it into an integral equation in $C_{h}(\mathbb{R})$ equipped with the standard supremum norm. Henceforth, we omit the dependence on the $x$-variable. The linear problem $\partial_{f} R=L_{1} R+f(t), R(0)=g$, can be solved by the semigroup $G(t)=e^{t+x^{2}}$. In Lemma 2.3 we prove that $G(t)$ is a uniformly bounded strongly continuous semigroup on $C_{h}(\mathbb{R})$. Thus, (2.2) transforms into

$$
R(t)=G(t) R(0)+\varepsilon^{2} \int_{0}^{t} G(t-s)[a(\varepsilon, s) R(s)+\varepsilon N(\varepsilon, s, R(s))+r(s)] d s .
$$

For each $D>0$, we have $\|N(\varepsilon, s, R)\| \leqq M$ for all $R$ with $\|R\| \leqq D$ and $\varepsilon \in\left(0, \varepsilon_{0}\right)$. With $\|G(s)\|,\|r\|,|a(s)| \leqq C$, we estimate

$$
\|R(t)\| \leqq 2 C d+\int_{0}^{t} \varepsilon^{2} C^{2}\|R(s)\| \mathrm{d} s+\varepsilon^{2} t C(\varepsilon M+C) .
$$


as long as $R(t)$ stays in the ball of radius $D$. With $\varepsilon^{2} t \leqq T_{0}$. Gronwall's inequality yields

$$
\|R(t)\| \leqq \bar{C} e^{\varepsilon^{2} C^{2}}, \quad \ddot{C}=2 C d+T_{0} C(\varepsilon M+C),
$$

for $t \leqq T_{0} / \varepsilon^{2}$. Let $\hat{C}=2 C d+T_{n} C(C+C)$ and $D=\hat{C} e^{c T_{i n}}$, and make $\varepsilon_{0}$ smaller, such that $\varepsilon M \leqq C$ (hence $\bar{C} \leqq \hat{C}$ ). Then (2.4) shows that $\|R(t)\| \leqq D$ for all $t \leqq T_{0} / \varepsilon^{2}$. The desired result now follows from $u(t, x)-u_{A}(t, x)=\varepsilon^{2} R(t, x)-$ $\varepsilon^{3}\left(A^{3} e^{3 x}+\right.$ c.c. $) / 64$.

LEMMA 2.3. The semigroup $G(t)=e^{L x}: C_{n}(\mathbb{R}) \rightarrow C_{b}(\mathbb{R}), t \geqq 0$, is uniformly bounded.

Proof. We use the representation of $G$ as a Dunford integral over the resolvent

$$
G(t)=\frac{1}{2 \pi i} \int_{\Gamma} e^{\lambda\left(L_{0}-\lambda\right)^{-1} d \lambda,}
$$

where $\Gamma \subset \mathbb{C}$ is a curve from $(-1+i) \infty$ to $(-1-i) \infty$ not intersecting $(-\infty, 0]$. The lemma is proved, if the resolvent $\left(L_{0}-\lambda\right)^{-1}$ exists in the whole sector $\mathbb{C}_{s}=\{\lambda \in \mathbb{C}: \lambda \neq 0,|\arg \lambda|<2 \pi / 3\}$ and satisfies

$$
\left\|\left(L_{0}-\lambda\right)^{-1}\right\| \leqq M /|\lambda| \quad \text { for all } \lambda \in \mathbb{C}_{S}
$$

(cf. [6, chap. IX.1]). For $\lambda \in \mathbb{C}_{S}$, we define $\omega=(-\lambda)^{1}$ with $\operatorname{Im} \omega>0$. Then, $L_{1} u-\lambda u=-\left(1+\partial_{x}^{2}+\omega\right)\left(1+\partial_{x}^{2}-\omega\right) u=f$ has the solution

$$
u=\left(L_{u}-\lambda\right)^{-1} f=-K_{0} \cdot{ }^{\circ} K_{a} f, \text { with } K_{a} g(x)=\int_{a} \frac{-1}{2 a} e^{-a|x-\xi|} g(\xi) d \xi,
$$

where $a_{ \pm}=(-1 \pm w)^{3}$ with $\operatorname{Re} a_{ \pm}>0$. Hence, the resolvent satisfies

$$
\left\|\left(L_{n}-\lambda\right)^{-1}\right\| \leqq \frac{1}{\left|a_{+}\right| \operatorname{Re} a_{+}\left|a_{-}\right| \operatorname{Re} a_{-}}=\frac{1}{\sqrt{|1+\lambda|}} \frac{1}{\operatorname{Re} a_{+} \operatorname{Re} a_{-}} .
$$

From $|\arg \lambda|<2 \pi / 3$, we find with $\rho^{2}=|\lambda|$ the estimates $|-1 \pm \omega| \geqq(1-\sqrt{3} \rho+$ $\left.\rho^{2}\right)^{1}$ and $|\arg (-1 \pm \omega)| \geqq \alpha(\rho)$, where $\tan (\pi-\alpha)=\rho /(1+\sqrt{3} \rho / 2)$ and $\alpha \epsilon$ $(2 \pi / 3, \pi)$. This implies Re $a_{ \pm} \geqq\left(1-\sqrt{3} \rho+\rho^{2}\right)^{\frac{1}{4}} \cos (\alpha(\rho) / 2)$. In both limits, $\rho \rightarrow 0$ and $\rho \rightarrow \infty$, we find $\sqrt{|1+\lambda|} \operatorname{Re} a_{+} \operatorname{Re} a_{-}>c \rho^{2}=c|\lambda|$, which is the desired estimate (2.5). Thus, the lemma is proved.

\section{The hyperbolic case}

We now consider a hyperbolic problem where the interest lies in the time- and space-dependent modulations of oscillations around a trivial state. As a model, we consider the Sine-Gordon equation (SGE)

$$
\partial_{1}^{2} u=\partial_{x}^{2} u-u+g(u), \text { where } g(u)=u-\sin u .
$$

Here $e^{i(k x-r u)}$ are the solutions of the linearised problem, where $k$ and $\omega$ are related by the dispersion relation $\omega^{2}=k^{2}+1$. The group velocity is given by $v=\partial \omega / \partial k=k / \omega$ and satisfies $|v|<1$. 
We are looking for modulated travelling waves in the form $u_{A}$ of (1.4), i.e. we define the slow time $T=\varepsilon^{2} t$ and the slow space variable $X=\varepsilon(x-v t)$ (cf. [1]). Guided by the proof of Theorem 2.1, we use the improved approximation

$$
v_{A}(t, x)=\varepsilon A(T, X) e^{t(k x-\omega t)}-\frac{1 / 6}{9 k^{2}-9 \omega^{2}+1} \varepsilon^{3} A(T, X)^{3} e^{3 t(k x-\omega t)}+\text { c.c. }
$$

Due to the correction term, we find the residuum

$$
\begin{aligned}
\rho(\varepsilon, t, x) & =\partial_{t}^{2} v_{A}-\partial_{x}^{2} v_{A}+v_{A}-g\left(v_{A}\right) \\
& =\varepsilon^{3}\left(-2 i \omega \partial_{T} A+\left(v^{2}-1\right) \partial_{X}^{2} A+\frac{1}{2}|A|^{2} A\right) e^{(k x-\omega t)}+\text { c.c. }+\sigma\left(\varepsilon^{4}\right)
\end{aligned}
$$

Hence, if $A$ satisfies the nonlinear Schrödinger equation (NLSE) (1.5), the residuum is $O\left(\varepsilon^{4}\right)$.

We have to be aware of the fact that the above estimates are pointwise in $(t, x) \in[0, \infty) \times \mathbb{R}$. However, for wave problems it is more convenient to work in energy space, namely $(u, \partial, u) \in Y=H^{\prime}(\mathbb{R}) \times L_{2}(\mathbb{R})$. It is well-known that, for any initial conditions in $Y$, the SGE has a unique solution $u=u(t, x)$ such that $t \in \mathbb{R} \rightarrow(u(t, \cdot), \partial, u(t, \cdot)) \in Y$ is continuous and bounded. Similarly, the NLSE has a unique solution $A=A(T, X)$ such that $T \in \mathbb{R} \rightarrow A(T, \cdot) \in Z=H^{1}(\mathbb{R})$ is continuous and bounded.

In constructing the approximate solution $v_{A}$ with a given solution $A$ of the NLSE, we have to recall the spatial scaling $X=\varepsilon(x-v t)$. Hence we obtain

$$
\left\|v_{A}(t, \cdot)\right\| \leqq 2 \varepsilon^{\frac{1}{2}}\left\|A\left(\varepsilon^{2} t, \cdot\right)\right\|+C \varepsilon^{2}\left\|A\left(\varepsilon^{2} t, \cdot\right)\right\|_{i}^{3} .
$$

Here and further on $\|\cdot\|$ denotes the $L_{2}$-norm and $\|\cdot\|_{1}$ the $H^{1}$-norm in the appropriate variable. For the residuum we have $\|\rho(\varepsilon, t, \cdot)\|=O\left(\varepsilon^{3}\right)$. Again we omit the $x$-dependence.

For the error we use the ansatz $\varepsilon^{\frac{3}{2}} R=u-v_{A}$ and obtain the equation

$$
\partial_{t}^{2} R=\partial_{x}^{2} R-R+\varepsilon^{2} a(\varepsilon, t) R+\varepsilon^{i} N(\varepsilon, t, R)+\varepsilon^{2} r(\varepsilon, t) .
$$

where $a(\varepsilon, t)=\left(1-\cos v_{A}(t)\right) / \varepsilon^{2}, r=\rho / \varepsilon^{3}$. To show that $N$ is $O(1)$, we use

$$
\begin{aligned}
N(\varepsilon, t, R) & =\varepsilon^{-4}\left[g\left(\varepsilon^{3} R+v_{A}\right)-g\left(v_{A}\right)-g^{\prime}\left(v_{A}\right) \varepsilon^{\frac{3}{3}} R\right] \\
& =\varepsilon^{-4} g^{\prime \prime}\left(v_{A}+\theta \varepsilon^{\frac{3}{3}} R\right) \varepsilon^{3} R^{2}=\frac{1}{\varepsilon} \sin \left(v_{A}+\theta \varepsilon^{\frac{1}{2}} R\right) R^{2},
\end{aligned}
$$

where $\theta \in[0,1]$ from the mean value theorem. Since $1 / \varepsilon \sin (\ldots)$ as well as $a(\varepsilon, t)$ act as multiplication operators in $L_{2}(\mathbb{R})$, their operator norm is the supremum norm. Hence, using $\left\|v_{A}\right\|_{x}=O(\varepsilon)$ and $\|R\|_{x} \leqq C\|R\|_{1}=O(1)$, we have the desired result.

Now, the analysis of Theorem 2.1 can be repeated by writing (3.2) as a first-order system for $(R, \partial, R)$ in the Banach space $Y$. The linear part is $\partial_{1}(R, S)=L(R, S)=\left(S, \partial_{x}^{2} R-R\right)$ and the associated semigroup $G(t)=e^{L^{\prime}}$ is an isometry for the norm $\|(R, S)\|_{Y}^{2}=\int_{\mathrm{R}}\left[\left(\partial_{x} R\right)^{2}+R^{2}+S^{2}\right] \mathrm{d} x$. Moreover, the nonlinear mapping $N$ is well defined from $Y$ into $L_{2}(\mathbb{R})$. Going through the estimates of the proof of Theorem 2.1 gives the following result:

Theorem 3.1. Let $A=A(T, X)$ be a solution of the NLSE such that the derivatives $\partial_{T}^{n} \partial_{X}^{k} A$ are in $C\left(\left[0, T_{1}\right], L_{2}(\mathbb{R})\right)$ for $n+k \leqq 2$ and let $u_{A}$ be the formal 
approximation (1.4). Then, for each $T_{0} \leqq T_{1}$ and each $d>0$, there exist $\varepsilon_{0}, C>0$ such that for all $\varepsilon \in\left(0, \varepsilon_{0}\right)$ the following statement holds. Let $u=u(t, x)$ be $a$ solution of the $S G E$ such that $\left\|\left(u(0), \partial_{,} u(0)\right)-\left(u_{A}(0), \partial_{1} u_{A}(0)\right)\right\|_{\gamma} \leqq d \varepsilon^{\prime} ;$ then the estimate

$$
\left\|\left(u(t), \partial_{1} u(t)\right)-\left(u_{A}(t), \partial_{1} u_{A}(t)\right)\right\|_{Y} \leqq C \varepsilon^{l} \text { for } t \in\left[0, T_{0} / \varepsilon^{2}\right]
$$

is satisfied.

We remark here that the theory cannot be carried out in spaces of functions which are bounded (e.g. quasi-periodic), since the semigroup $G(t)$ of the dispersive wave operator is not uniformly bounded on spaces like $C_{b}(\mathbb{R})$.

\section{Discussion and generalisations}

From the proof of Theorem 2.1 we see that the result can be generalised in several directions. Firstly, we may increase the order of accuracy of the formal solution $v_{A}$. This can be done as described in [11] or [4]. Having the residuum $\rho=\partial_{1} v_{A}-L_{\varepsilon} v_{A}+v_{A}^{3}$ of order $O\left(\varepsilon^{n}\right)$ with $n>4$, we introduce the scaling $R=\varepsilon^{2-n}\left(u-v_{A}\right)$ and again obtain the same equation (2.2) but with $\varepsilon^{n-1}$ as a factor of $N(\varepsilon, \ldots)$. Again we obtain $|R(t, x)|=O(1)$ on the time interval $\left[0, T_{n} / \varepsilon^{2}\right]$, and thus $\left|u(t, x)-v_{A}(t, x)\right| \leqq C \varepsilon^{n-2}$. A similar result holds in the hyperbolic case.

Secondly, we can treat arbitrary nonlinearities $g$ with $g(u)=Q\left(|u|^{3}\right)$ instead of $u^{3}$ or $u-\sin u$. Moreover, in the parabolic case $g$ could also depend on the derivatives $\partial_{x}^{k} u, k=1,2,3$. Then the smoothing properties of the semigroup have to be exploited, see $[2,11]$. Moreover, an explicit $t$ - and $x$-dependence for $g$ can be allowed, as long as this dependence is uniform and we are able to construct approximate solutions which have a sufficiently small residuum.

The case of a quadratic leading term in the nonlinearity is explicitly excluded, since there the linear perturbation term $g^{\prime}\left(v_{A}(t, x)\right) R$ would only be of order $O(\varepsilon)$. An error estimate would only be possible on the shorter time-scale $1 / \varepsilon$, which is not the natural time-scale for the modulations. For the quadratic case, the only rigorous results over the correct time scale are given in [11].

Thirdly, we note that the method is simple enough to allow for the case when $u$ is vector-valued, in particular for studying partial differential equations on cylindrical domains, where $u(t, x)$ can be thought of as having values in a Banach space. We leave this for future research.

From the integral equation (2.3), we can also prove the existence of the solution $R$ by the standard contraction mapping principle. Note that the Lipschitz constant is small even over time intervals of length $T_{0} / \varepsilon^{2}$, due to the factor $\varepsilon^{\alpha}$ with $\alpha>0$ in front of $N(\varepsilon, t, R)$. This observation is helpful in problems where the full system does not guarantee global existence for all initial data. Then the solutions with initial conditions of modulation type, i.e. as in (1.2), exist as long as predicted by the associated modulation equation, while general initial conditions might lead to blow up in finite time, independently of $\varepsilon$. 


\section{Acknowledgments}

The authors are grateful for stimulating discussions with W. Eckhaus and $\mathrm{A}$. van Harten. The research was partially supported by Deutsche Forschungsgemenischaft (DFG) under grant $\mathrm{Ki} 131 / 5-1$, by Land Baden-Württemberg under grant $7532.273-3 / 1$, and by the European Community under ST2J-0316-C (EDB).

\section{References}

I F. Calogero and W. Eckhaus. Nonlinear evolution equations, rescalings, model PDEs and their integrability: I + IJ. Inverse Problems 3 ( 1987), 229-262; 4 ( 1988) II-33.

2 P. Collet and J.-P. Eckmann. The time dependent amplitude equation for the Swift-Hohenberg problem. Comm. Math. Physics 132 (1990), 139-I53.

3 R. C. DiPrima, W. Eckhaus and L. A. Segel. Non-linear wave-number interaction in near-critıcal two-dimensional flows. J. Flund Mech. 49 (197I), 705-744.

4 G. Jooss, A. Mielke and Y. Demay. Theory of steady Ginzburg-Landau equation in hydrodynamic stability problems. European J. Mech. B/Fluids 3 (1989), 229-268

5 L. A. Kalyakin. Long wave asymptotics, integrable equations as asymptotic limits of non-linear systems. Russ. Math. Surveys 44 (1) (1989), 3-42.

6 T. Kato. Perlurbation Theory for Linear Operalors (Berlın: Sprınger, 1966).

7 M. S. Krol. On a Galerkin-averaging method for weakly nonlinear wave equations. Math. Methods Applied Sci. 11 (1989), 649-664.

8 A. Mielke. Reduction of PDEs on domains with several unbounded directions J. Appl. Malh Phys. (ZAMP) 43 (1992).

9 A. Newell and J. Whitehead. Finıte bandwidth, finite amplıtude convection. J. Flurd Mech. 38 (1969), 279-303.

10 A. Stroucken and F. Verhulst. The Galerkin-averaging method for nonlinear, undamped continuous systems. Math. Methods Applied Scl. 9 (1986), 520-549.

II A. van Harten. On the validity of Ginzburg-Landau's equation. J. Nonlinear Science 1 (I99I), $397-422$. 